\title{
Irrigación del nervio isquiático en caninos
}

\author{
Llano, E.G.; F. Quintana, C.; Baez, A.D.; Cabrera, W.R.; Benítez, J.S. \\ Cátedra de Anatomía II y Cátedra de Histología y Embriología, Facultad de Ciencias Veterinarias, UNNE, \\ Sargento Cabral 2139, Corrientes (3400), Argentina. E-mail: anato2@vet.unne.edu.ar
}

\begin{abstract}
Resumen
Llano, E.G.; F. Quintana, C.; Baez, A.D.; Cabrera, W.R.; Benítez, J.S.: Irrigación del nervio isquiático en caninos. Rev. vet. 28: 1, 14-18, 2017. El objetivo del trabajo fue determinar origen y distribución segmentaria de los vasos sanguíneos que constituyen el sistema extrínseco en la irrigación del nervio isquiático del perro. Se trabajó con 30 miembros pelvianos previamente inyectados con pasta de repleción vascular (látex coloreado). Posteriormente a la disección el material se conservó por inmersión en una solución de formol al $10 \%$. De las observaciones registradas surge que el segmento proximal del nervio isquiático a nivel de la fosa trocantérica del fémur esta irrigado por ramas de la arteria glútea caudal. Por su parte, el segmento distal en el nivel del tercio medio y distal del fémur, está irrigado por ramas de la arteria femoral caudal distal. La existencia de esta irrigación debe tenerse en cuenta al momento de efectuar maniobras quirúrgicas y traumatológicas en esta especie.
\end{abstract}

Palabras claves: canino, anatomía, nervio isquiático, angiología.

\begin{abstract}
Llano, E.G.; F. Quintana, C.; Baez, A.D.; Cabrera, W.R.; Benítez, J.S.: Irrigation of the sciatic nerve in dogs. Rev. vet. 28: 1, 14-18, 2017. The objective was to determine origin and segmental distribution of blood vessels that constitute the extrinsic system in the irrigation of the dog sciatic nerve. We worked with 30 pelvic limbs previously injected with colored latex. After the dissection material was preserved by immersion in a solution of $10 \%$ formalin. Findings show that the proximal segment of the sciatic nerve at the trochanteric fossa of the femur is supplied by branches of the caudal gluteal artery. The distal region at the middle and distal thirds of the femur is supplied by branches of the distal caudal femoral artery. This irrigation characteristic should be considered when making surgical and orthopedic maneuvers in this species.
\end{abstract}

Key words: dog, anatomy, sciatic nerve, angiology.

\section{INTRODUCCIÓN}

Las lesiones en los nervios periféricos determinan la parálisis de la musculatura que recibe sus impulsos o la pérdida de sensibilidad en la zona de referencia. Por ello, su reparación es practicada desde hace mucho tiempo, desarrollándose gran número de estudios en el campo experimental ${ }^{2,9,11,15}$.

Antiguamente se creía que los nervios periféricos no tenían capacidad para regenerarse después de una lesión. Posteriormente, en los monogenistas aparece el concepto de regeneración del segmento proximal de la fibra por la conexión de axón al cuerpo neuronal. Sin embargo, para los poligenistas, eran las porciones distales de los axones las que permanecían viables y se re-anastomosaban con el segmento proximal ${ }^{13}$.

Recibido: 28 setiembre 2016 / Aceptado: 12 diciembre 2016
Actualmente se considera que el sistema nervioso periférico es capaz de una regeneración completa luego de un trauma menor. El sistema nervioso central relegó su capacidad de reparación a favor de la mayor complejidad sináptica y la integración de redes neuronales, mientras que el sistema nervioso periférico, mucho más expuesto, mantuvo la habilidad de regenerarse y restaurar sus conexiones apropiadas ${ }^{8,12}$.

En el perro, las lesiones del nervio isquiático son frecuentes. Las fracturas en el hueso ileon, fracturas proximales del fémur o las compresiones tumorales pueden dañarlo. Los procedimientos quirúrgicos de la cadera o el fémur, las reparaciones de la seudohernia perineal o las mismas inyecciones intramusculares en los músculos caudales del muslo pueden lesionar este nervio ${ }^{8}$. Frecuentemente los accidentes automovilísticos o las mordeduras tienen consecuencias importantes sobre el nervio isquiático ${ }^{5,23,25}$. 
Cuando el nervio isquiático se encuentra lesionado ocurre la parálisis de grupos musculares que contribuyen a la extensión de la cadera y a la flexión de la rodilla. El miembro afectado se apoya sobre el dorso de los dedos y el tarso se flexiona y extiende pasivamente cuando el peso se recarga sobre él. Hay pérdida de la sensibilidad en diferentes grados ${ }^{8}$.

Varios autores destacan la necesidad de disponer de abundante información anatómica para avanzar con las técnicas de microcirugías reparadoras. Algunos advierten sobre la necesidad de preservar la irrigación ${ }^{19}$, otros recalcan que el conocimiento de la estructura de un nervio -en cada uno de sus tramos- adquiere importancia en los procedimientos utilizados en las estimulaciones con fines terapéuticos ${ }^{6}$.

La funcionalidad de la regeneración de nervios periféricos depende de una adecuada vascularización que asegure una óptima oxigenación y la presencia de células no neuronales, factores de crecimiento, moléculas de adhesión y materiales de la matriz extracelular ${ }^{11,20}$. En anatomía humana existen amplios conocimientos sobre el origen de los vasos sanguíneos que constituyen el sistema extrínseco de los troncos nerviosos periféricos.

El aporte sanguíneo de un nervio periférico lo constituye un plexo vascular complejo, compuesto por dos sistemas arteriales uno extrínseco y otro intrínseco anastomosados entre sí. Los sistemas arteriales son tortuosos para permitir el desplazamiento longitudinal amplio del nervio, previa constricción a la tensión ejercida con los cambios posturales ${ }^{13}$.

El sistema extrínseco se localiza en la superficie del nervio manteniendo una posición relativamente constante a lo largo de toda su longitud, aportando de forma mesoneural arterias nutricias que varían en tamaño y número, penetrando en el nervio a intervalos irregulares ${ }^{13}$. El sistema extrínseco se origina de las arterias vecinas, vasos periósticos y musculares adyacentes más pequeños. Las ramas de estos vasos se dividen en ascendentes y descendentes cuando alcanzan el epineuro y se anastomosan con el sistema intrínseco ${ }^{12,22}$.

A inicios del Siglo XXI, utilizando una clasificación preexistente sobre las configuraciones del pedículo vascular dominante, se reconocieron cinco tipos de aporte vascular al nervio periférico: A, ideal para injertos, con pocas ramas colaterales y que recibe vascularización de una arteria única; $\mathrm{B}$, difiere del anterior en el número de colaterales del tronco nervioso; $\mathrm{C}$, comprende varios troncos nerviosos con un vaso nutricio único; D, tronco nervioso con arteria nutricia propia con calibre insuficiente para sutura microquirúrgica; y E, tronco nervioso con colaterales y diferentes arterias ${ }^{16}$.

Las arterias responsables de la irrigación del plexo lumbar provienen en forma constante de las arterias lumbares, de la arteria iliolumbar, rama del tronco posterior de la arteria iliaca interna (hipogástrica) y de la arteria iliaca externa a través de la arteria circunfleja iliaca profunda. Las ramas arteriales muy delgadas llegan ya sea en forma directa o acompañando un largo trecho adheridas al perineuro antes de penetrarlo ${ }^{24}$.
El sistema intrínseco está conformado por los plexos epineural, perineural, endoneural y sus vasos comunicantes ${ }^{12,22}$. Los capilares endoneurales presentan las mismas características estructurales y funcionales de los capilares del sistema nervioso central, representando una prolongación de la barrera vascular cerebral ${ }^{13,18}$.

Para una función adecuada se requiere una conexión anatómica y fisiológica con el sistema nervioso central y un suministro continuo y conveniente de oxígeno a través del sistema vascular extrínseco e intrín$\operatorname{seco}^{21}$.

En el caso particular del nervio isquiático del canino, no se encuentran trabajos que describan la arteria principal o colateral de la que se desprenden los demás vasos, cómo y dónde se distribuyen, recorren y penetran al tronco nervioso. Esta vascularización debe ser considerada en la longitud total del nervio y para cada segmento en particular, determinando de esta manera la presencia más frecuente de estos vasos sanguíneos y la probable aparición de variaciones.

\section{MATERIAL Y MÉTODOS}

Se utilizaron 15 cadáveres caninos, donados por el Corralón Municipal de la Dirección de Zoonosis de la Municipalidad de la Ciudad de Corrientes, Argentina, Hospital de Clínicas de la Facultad de Ciencias Veterinarias de la UNNE y clínicas privadas.

Se ejecutó la siguiente metodología: laparotomía ventral mediana con desplazamiento lateral de las vísceras de la cavidad abdominal. Localización y canalización de la arteria aorta abdominal en la región de las arterias renales. Inyección de la pasta de repleción vascular utilizando látex coloreado. Reposo de $48 \mathrm{~h}$ en freezer, a temperatura de congelación, logrando que el látex coloreado adquiera su consistencia sólida y la conservación del cadáver en ese tiempo.

Concluido el lapso de reposo, el material se retiró del freezer, hasta su descongelado, para luego efectuar la evisceración y sección del tren pelviano en la región lumbar. Se realizó la disección clásica del tronco nervioso y con la ayuda de una lupa se determinaron los vasos sanguíneos constituyentes del sistema extrínseco de irrigación, visualizando el origen de los mismos y su distribución en el segmento proximal a nivel de la fosa trocantérica del fémur y en el segmento distal a nivel del tercio medio del mismo hueso, en proximal a la división del tronco principal.

Se efectuó la documentación fotográfica de las disecciones. Se esquematizaron las observaciones registradas. Los preparados anatómicos se conservaron por inmersión en una solución de formol al 10\%.

\section{RESULTADOS}

En todos los miembros estudiados se observó que el nervio isquiático no fue acompañado paralelamente por una sola arteria en todo su recorrido; sin embargo, en las disecciones se distinguieron arterias que lo cru- 


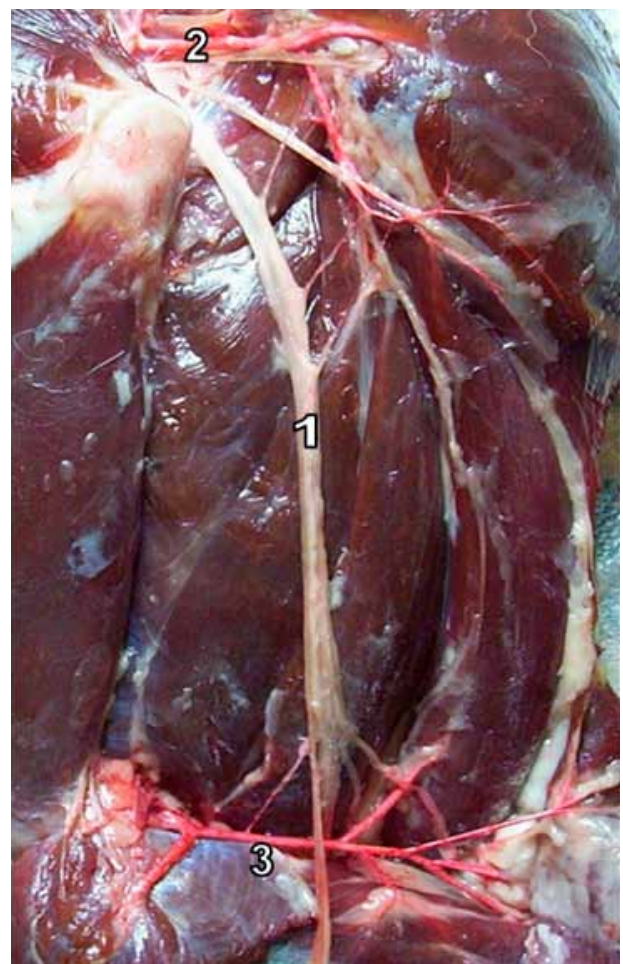

Figura A. 1: nervio isquiático, 2: arteria glútea caudal, 3: arteria femoral caudal distal.

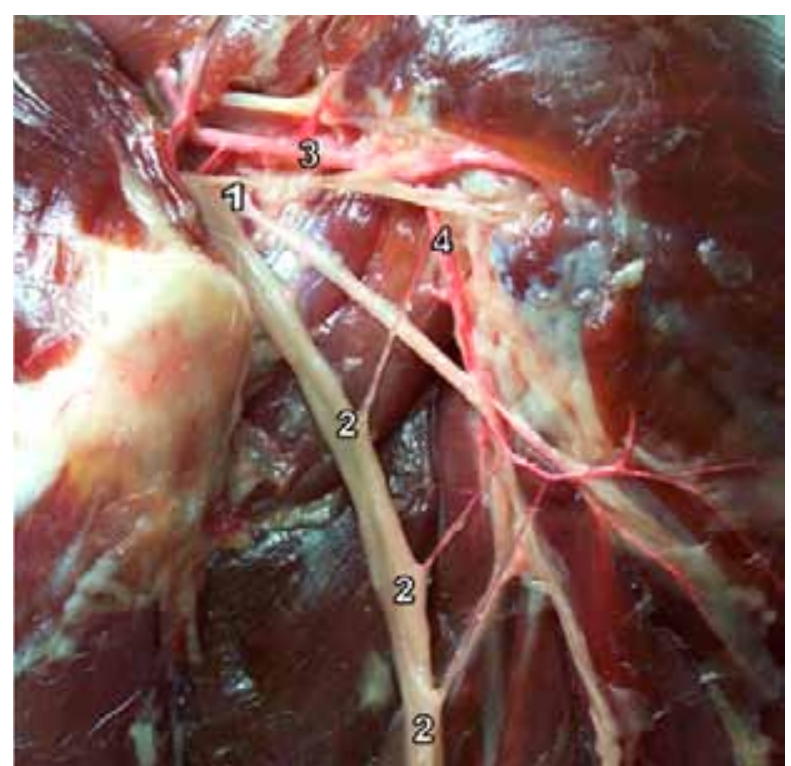

Figura B. 1: vasos directos de la arteria glútea caudal, 2: vasos de la rama muscular, 3: arteria glútea caudal, 4: rama muscular de 3 .

zan. Por este motivo el sistema vascular extrínseco del nervio isquiático se desprende de vasos que cruzan su trayecto en cercanía a los segmentos proximal y distal considerados en este estudio (Figura A).

La irrigación del segmento proximal del nervio isquiático se realizó a través de dos a cinco pequeños vasos arteriales descendentes y flexuosos, cubiertos por tejido adiposo. Ellos se originaron directamente de la arteria glútea caudal cuando ésta acompañó al nervio, o de las ramas musculares para los músculos semitendinoso y semimembranoso, cuando la arteria glútea caudal se encontraba alejada. Estos pequeños vasos arteriales alcanzaban al nervio por el epineuro y luego de dividirse en cortos trayectos ascendentes y descendentes se continuaron como sistema vascular intrínseco (Figura B).

El segmento distal del nervio isquiático estaba irrigado por uno o dos pequeños vasos arteriales ascendentes que se originaban directamente de la arteria femoral caudal distal o de la rama muscular para el músculo semimembranoso. Estos pequeños vasos alcanzaban a los nervios peroneo y tibial con una distribución similar al segmento proximal (Figuras C y D).

\section{DISCUSIÓN}

Los nervios están asociados a una red vascular, dado que la conducción nerviosa es un proceso que

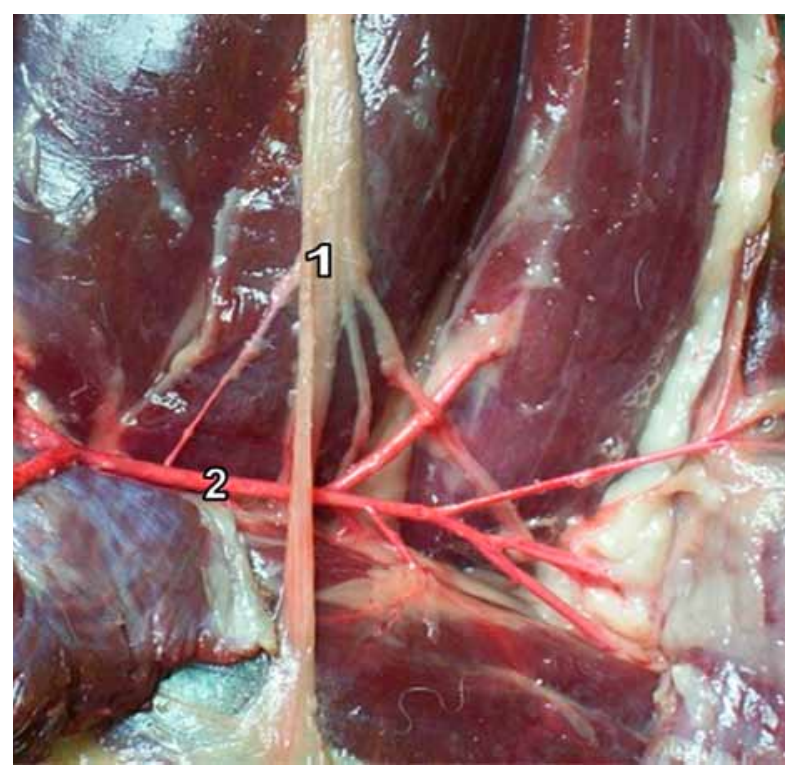

Figura C. 1: vaso directo de la arteria femoral caudal distal, 2: arteria femoral caudal distal.

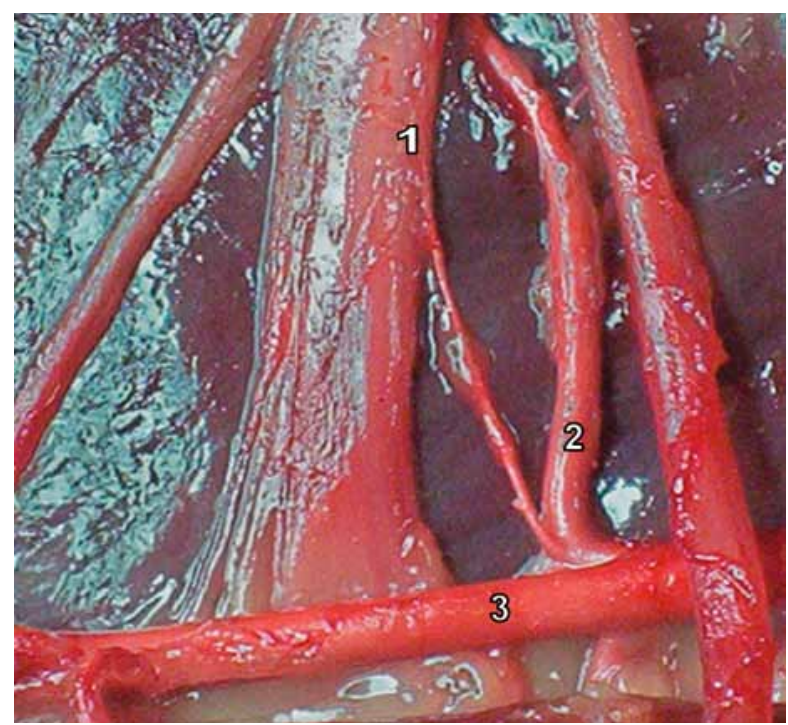

Figura D. 1: vaso de la rama muscular, 2: rama muscular de la arteria femoral caudal distal, 3: arteria femoral caudal distal. 
consume energía. Desde un inicio, los primeros trabajos experimentales vincularon los desórdenes de los nervios periféricos con la vascularización y es relativamente sencillo entender la vulnerabilidad de los axones a la isquemia por la gran distancia que tienen del cuerpo neuronal ${ }^{17}$. Los problemas vasculares son responsables de varios fenómenos clínicos como parálisis flácida o desórdenes de sensibilidad, llamados en conjunto neuropatías isquémicas ${ }^{1}$.

Para asegurar la adecuada irrigación sanguínea, los nervios periféricos tienen dos sistemas vasculares que se anastomosan entre sí, denominados extrínseco e intrínseco. El primero está formado por arterias, arteriolas y vénulas que llegan hasta el epineuro. El número de estos vasos arteriales es variable entre los individuos y proceden de las arterias principales o de sus ramas. En general, los nervios de mayor tamaño no necesariamente reciben más vasos ${ }^{17}$.

En anatomía humana existen amplios conocimientos sobre el origen de los vasos sanguíneos que constituyen el sistema extrínseco de los troncos nerviosos periféricos. En el caso particular del nervio isquiático en caninos no se encuentran descripciones sobre la arteria principal o colateral de la que se desprenden los vasos, cómo y dónde se distribuyen, recorren y penetran al tronco nervioso. El sistema extrínseco de vascularización de los nervios periféricos puede clasificarse en aquéllos que tienen una arteria dominante que corre a lo largo del nervio y aquéllos que tienen dos o más arterias que entran al nervio en diferentes puntos ${ }^{4}$. El nervio isquiático estudiado en este trabajo pertenece a este último grupo.

Observaciones efectuadas por nuestro grupo de trabajo indicaron que el nervio tibial, el nervio femoral, el segmento proximal de los nervios cubital y mediano del perro y los nervios palmares y plantares del equino, están acompañados en su recorrido por una arteria de grueso calibre a partir de la cual se originan los vasos que constituyen el sistema extrínseco. Al igual que en el nervio radial, en este trabajo se observó que el sistema extrínseco de vascularización del nervio isquiático en el segmento proximal se origina de ramas que salen directamente de una arteria principal, en este caso de la arteria glútea caudal o colaterales musculares para los músculos semitendinoso y semimembranoso. Son dos a cinco pequeños vasos arteriales descendentes y flexuosos, cubiertos por tejido adiposo, lo que permite el desplazamiento longitudinal amplio del nervio ante los cambios posturales ${ }^{13}$.

En el segmento distal del nervio isquiático se encontraron uno o dos pequeños vasos arteriales ascendentes que se originaron directamente de la arteria femoral caudal distal o de la rama muscular para el músculo semimembranoso. Estos pequeños vasos alcanzaron a los nervios peroneo y tibial, los recorrieron hacia proximal y cuando llegaron al nervio isquiático se continuaron en forma intraneural.

En algunos trabajos se observa que el sistema extrínseco tiende a mantenerse en una posición relativa- mente constante a lo largo de toda su longitud, aportando de forma mesoneural arterias nutricias que penetran al nervio ${ }^{13}$. El sistema extrínseco del nervio isquiático en el perro no mantiene esa constancia en la superficie a lo largo de toda su longitud. En el segmento proximal las arterias nutricias son más numerosas y de menor diámetro que en el segmento distal.

Aunque en la formación del sistema extrínseco pueden participar arterias vecinas, así como pequeños vasos periósticos y musculares adyacentes ${ }^{12,22}$, en los casos observados en este trabajo dicho sistema se originó de arterias destinadas a la irrigación muscular.

Los vasos sanguíneos ascendentes y descendentes llegan al nervio y recorren el epineuro en una distancia variable antes de penetrarlo ${ }^{12,22}$. En el plexo lumbar lo hacen en forma inmediata o acompañan un largo trecho adheridos al perineuro antes de penetrarlo ${ }^{24}$. En este trabajo, posteriormente a la división ascendente y descendente, en el segmento proximal la penetración al nervio fue más inmediata, con escaso recorrido por el epineuro, mientras que en el segmento distal el recorrido por el epineuro fue mayor, para continuarse en forma intraneural constituyendo el sistema intrínseco.

No solo el funcionamiento normal, sino también la regeneración de nervios periféricos, dependen de una adecuada vascularización que asegure oxigenación, así como la presencia de células no neuronales, factores de crecimiento y moléculas de adhesión, entre otros ${ }^{20}$. Las técnicas de sutura para el mejor afrontamiento fascicular e injertos nerviosos consideran vasos y calibre de los fascículos ${ }^{3}$.

La isquemia es perjudicial al crecimiento axonal y en la valoración del daño estructural de un nervio lesionado está recomendado tener prudencia en la preservación de la irrigación, aún cuando la circulación colateral del tronco nervioso puede permitir un mayor margen antes de dañar la circulación ${ }^{7,22}$.

Anteriormente, ya se observaba que la sutura epineural de los nervios periféricos no conseguían una recuperación funcional satisfactoria en gran número de casos debido al aporte sanguíneo insuficiente ${ }^{10}$; la revascularización nerviosa luego de una sutura puede ser promovida con la reparación de los tejidos adyacentes ${ }^{14}$. En este caso si la conexión con el sistema nervioso central no se ve alterada, la función del nervio isquiático en el perro está asegurada por un sistema extrínseco de vascularización en el segmento proximal, con mayor número de arterias y con menor número de arterias pero éstas de mayor diámetro en el segmento distal.

El nervio isquiático, por sus características funcionales y por la configuración de su pedículo vascular dominante, pertenece a la categoría $\mathrm{E}$ anteriormente mencionada ${ }^{16}$, por lo cual está considerado como el menos indicado para ser utilizado como donante de injertos.

El sistema extrínseco de vascularización se comporta de manera diferente en los segmentos estudiados. El segmento proximal está irrigado por varias arterias, ninguna de ellas con un diámetro suficiente como para 
considerarla dominante. La irrigación del segmento distal está asegurada por arterias dominantes que recorren en forma ascendente a los nervios tibial y peroneo, para luego alcanzar al nervio isquiático en forma intraneural.

\section{REFERENCIAS}

1. Adams WE. 1942. The blood supply of nerves: I. Historical review. J Anat 76: 323-341.

2. Avala GH, Teijeira FM, Beguiristáin FL, Imízcoz FL, Cañadel FM. 1973. Distintos tipos de reparación quirúrgica en nervios periféricos. Rev Med Univ Navarra 17: 71-77.

3. Bazaldúa JJ, Quiroga A, Velázquez SE, Villarreal EE, Tijerina O, Elizondo RE, Garza O, Guzmán S. 2011. Estudio de la morfología vascular en los nervios de la extremidad inferior utilizados como injertos. Rev Investig Clin 63: 461-466.

4. Breidenbach W, Terzis JK. 1984. The anatomy of free vascularized nerve grafts. Clin Plast Surg 11: 65-71.

5. Chrisman CL. 1996. Neuropatías periféricas. En: Fisiopatología y clínica quirúrgica en animales pequeños (Bojrab MJ, ed.), Intermédica, Buenos Aires, p. 1192-1206.

6. Del Sol M, Olave E, Contreras J, Vásquez B. 2010. Morfometría de los nervios isquiático, tibial y fibular común en el hombre. Int J Morphol 28: 385-388.

7. Freinkel F, Orta R. 2009. Técnicas microquirúrgicas usadas en la reparación de los nervios periféricos Rev Arg Neurocir 23: 4 (www.scielo.org.ar/scielo.php?script)

8. Garibaldi L. 2003, Monoparesias y monoplejias. En: El libro de neurología para la práctica clínica (Pellegrino F, Suraniti A, Garibaldi L eds), Intermédica, Buenos Aires, p. 161-167.

9. Garrido J, Hernández P, Carriel S, García S, Sáez JA, Alaminos M, Torres J. 2012. Tratamiento de las lesiones de los nervios periféricos. Tendencias actuales del tratamiento quirúrgico. Actual Med 97: 45-55.

10. Hasse J, Bjerre P, Semesen K. 1984. Median and ulnar nerve transactions treated with microsurgical interfascicular cable grafting with autogenous sural nerve. J Neurosurg 53: 73-84.

11. Leal L, Perdomo S, Spinel C. 2004. Aislamiento y cultivo de fibroblastos endoneurales. Acta Biol Colomb 9: 57-65.

12. Lühers D, Riet G, Pioli MF. 2003. Citología del sistema nervioso periférico y su relevancia en las enfermedades neurológicas. En: El libro de neurología para la práctica clínica (Pellegrino F, Suraniti A, Garibaldi L, eds), Intermédica, Buenos Aires, p. 622-626.
13. Marin M, Castello JR, Santos HX. 2001. Lesiones agudas de los nervios periféricos. www.secpre.org/documentos

14. Merle M, Dautel G. 1991. Vascularised nerve grafts. $J$ Hand Surg 16: 483-488.

15. Padilla FJ, Mendoza F, Villarreal JL, Acosta JR. 2015. Reparación secundaria de nervio mediano con injerto autógeno de nervio sural. Rev Esp Méd Quir 20: 328-331.

16. Pérez R, Labrador JM, Lara F, Vivas AM, Hijano JC, Martinez R., Cabrera M. 2001. Injertos (cutáneos, dermograsos, tendinosos, nerviosos, óseos, cartilaginosos y vasculares). www.secpre.org/documentos

17. Reina MA, Lopez A, Villanueva MC, De Andres JA, León GI. 2000. Morfología de los nervios periféricos, de sus cubiertas y de su vascularización. Rev Esp Anest \& Reanim 50: 80-86.

18. Reina MA, Lopez A, Villanueva MC, De Andres JA, Maches F. 2003. La barrera hemato-nerviosa en los nervios periféricos. Rev Esp Anest \& Reanim 47: 464-475.

19. Restrepo Y. 2005. Injertos nerviosos de tubos perineurales vascularizados en la reparación de nervios. http:// www.encolombia.com/orto11397injertos1.htm

20. Rosales M, Peregrina J, Hernández J, Nolasco G, Chávez ME, Gómez U, Albarrán E. 2008. Regeneración del nervio ciático axotomizado del perro con prótesis de quitosana precargadas con pregnenolona. Vet Méx 39: 5566.

21. Rodkey WG. 1993. Peripheral nerve surgery. En: Textbook of small animal surgery (Slatter D ed), Saunders Co. p. 1135-1141.

22. Shores A. 2001. Sistema nervioso y órganos de la sensación. En: Técnicas actuales en cirugía de pequeños animales (Bojrab MJ, ed), Intermédica, Buenos Aires, p. 67-75.

23. Swaim SF. 1980. Sistema nervioso periférico. En: Medicina y cirugía en especies pequeñas (Bojrab MJ, ed), Edit. Continental, México, p. 29-33.

24. Terraes AR, De los Reyes MR, Gallovich JM, Recalde F, Romero E. 2001. Irrigación del plexus lumbalis. Comunicaciones Cientificas y Tecnológicas 2001. http://www1. unne.edu.ar/cyt/2001/3-Medicas/M-042.pdf

25. Ushikoshi WS. 2003. Síndromes neuropáticos. En: El libro de neurología para la práctica clínica (Pellegrino F, Suraniti A, Garibaldi L ed), Intermédica, Buenos Aires, p. 203-217. 